\title{
SOLUTIONS FOR THE FRACTIONAL $p$-LAPLACIAN SYSTEMS WITH SEVERAL CRITICAL SOBOLEV-HARDY TERMS
}

\author{
IRAJ DEHSARI AND NEMAT NYAMORADI*
}

Abstract. In this paper, we consider a class of fractional $p$-Laplacian system with three fractional critical Sobolev-Hardy exponents. By the Ekeland variational principle and the MountainPass theorem, we study the existence and multiplicity of positive solutions to the system.

Mathematics subject classification (2010): 35B33, 35J60, $35 \mathrm{~J} 65$.

Keywords and phrases: Fractional $p$-Laplacian, variational method, critical Hardy-Sobolev exponent, concentration-compactness principle.

\section{REFERENCES}

[1] C. Alves, D. Filho And M. Souto, On systems of elliptic equations involving subcritical or critical Sobolev exponents, Nonlinear Anal., 42, (2000), 771-787.

[2] V. Ambrosio, Ground states for a fractional scalar field problem with critical growth, Differential Integral Equations, 30, (1-2) (2017), 115-132.

[3] V. Ambrosio, Periodic solutions for critical fractional problems, Calc. Var. Partial Differential Equations, 57, (2) (2018), Art. 45, 31 pp.

[4] B. Barrios, E. Colorado, R. Servadei And F. Soria, A critical fractional equation with concave-convex power nonlinearities, Ann. Inst. H. Poincaré Anal. Non Linéaire, 32, (4) (2015), 875900.

[5] X. CABré And J. TAN, Positive solutions of nonlinear problems involving the square root of the Laplacian, Adv. Math., 224, (5) (2010), 2052-2093.

[6] W. Chen, Fractional elliptic problems with two critical Sobolev-Hardy exponents, Elect. J. Differ. Equat., 2018, (22) (2018), 1-12.

[7] W. Chen, S. Deng, The Nehari manifold for a fractional p-Laplacian system involving concaveconvex non-linearities, Nonlinear Anal, 27, (2016), 80-92.

[8] W. Chen AND Y. GuI, Multiple solutions for a fractional p-Kirchhoff problem with Hardy nonlinearity, Nonlinear Anal., 188, (2019), 316-338.

[9] W. Chen, S. Mosconi And M. SQuassina, Nonlocal problems with critical Hardy nonlinearity, J. Funct. Anal., 275, (11) (2018), 3065-3114.

[10] K. Cheng AND L. WANG, Non-homogeneous problem for fractional Laplacian involving critical Sobolev exponent, Electron. J. Differential Equations 2017, Paper No. 304, 24 pp.

[11] Z. Deng AND Y. HUANG, Existence and multiplicity of symmetric solutions for semilinear elliptic equations with singular potentials and critical Hardy-Sobolev exponents, J. Math. Anal. Appl., 393, (2012), 273-284.

[12] J. M. do Ó, J. Giacomoni And P. K. Mishra, Nehari Manifold for Fractional Kirchhoff Systems with Critical Nonlinearity, Milan J. Math., 85, (2018), 127-150.

[13] A. Fiscella, G. Molica Bisci And R. Servadei, Multiplicity results for fractional Laplace problems with critical growth, Manuscripta Math., 155, (3-4) (2018), 369-388.

[14] A. FISCELla AND P. PUCCI, p-Fractional Kirchhoff equations involving critical nonlinrarities, Nonlinear Anal. RWA, 35, (2017), 350-378.

[15] Z. Guo, S. Luo And W. Zou, On critical systems involving fractional Laplacian, J. Math. Anal. Appl., 446, (1) (2017), 681-706. 
[16] X. He, M. SQuassina AND W. Zou, The Nehari manifold for fractional systems involving critical nonlinearities, Commun. Pure Appl. Anal., 15, (4) (2016), 1285-1308.

[17] Q. Hong AND Y. YANG, Existence and multiplicity of solutions to the fractional p-Laplacian system involving critical Hardy-Sobolev exponents, (2019), doi:10.13140/RG.2.2.12932.88968.

[18] A. Iannizzotto, S. Liu, K. Perera And M. Squassina, Existence results for fractional $p$ Laplacian problems via Morse theory, Advances in Calculus of Variations, 9, (2) (2014), 101-125.

[19] A. IAnNizzotto AND M. SQuassina, Weyl-type laws for fractional p-eigenvalue problems, Asymptotic Anal., 88, (2014), 233-245.

[20] Y. LI, Q. GUO AND P. NIU, The existence of solutions for quasilinear elliptic problems with combined critical Sobolev-Hardy terms, J. Math. Anal. Appl., 388, (2012), 525-538.

[21] E. Lindgren And P. Lindqvist, Fractional eigenvalues, Calc. Var. Partial Differential Equations, 49, (2014), 795-826.

[22] P. L. Lions, The concentration-compactness principle in the calculus of variations, the limit case, part 1, Rev. Mat. Iberoam., 1, (1985), 145-201.

[23] S. MARANO AND S. Mosconi, Asymptotic for optimizers of the fractional Hardy-Sobolev inequality, Commun. Contemp. Math., (2018), 1850028.

[24] J. Mawhin And M. Willem, Critical Point Theory and Hamiltonian Systems, Appl. Math. Sci. 74, Springer, New York 1989.

[25] G. Molica Bisci, V. RǍdulescu And R. ServadeI, Variational Methods for Nonlocal Fractional Problems, with a Foreword by Jean Mawhin, Encyclopedia of Mathematics and its Applications, Cambridge University Press, 162 Cambridge, 2016.

[26] G. Palatucci And A. Pisante, Improved Sobolev embeddings, profile decomposition, and concentration-compactness for fractional Sobolev spaces, Calc. Var. Partial Differential Equations, 50, (3-4) (2014), 799-829.

[27] H. RABINOwITZ, Methods in critical point theory with applications to differential equations, Amer. Math. Soc, CBMS, 1986.

[28] R. Servadei And E. VAldinoci, The Brezis-Nirenberg result for the fractional Laplacian, Trans. Amer. Math. Soc., 367, (1) (2015), 67-102.

[29] S. SHAKERIAN, Multiple positive solutions for Nonlocal elliptic problems involving the Hardy potential and concave-convex nonlinearities, (2018), arXiv:1708.01369v1.

[30] J. TAN, The Brezis-Nirenberg type problem involving the square root of the Laplacian, Calc. Var. Partial Differential Equations, 42, (1-2) (2011), 21-41.

[31] M. Xiang, B. Zhang AND X. Zhang, A nonhomogeneous fractional p-Kirchhoff type problem involving critical exponent in $\mathbb{R}^{N}$, Adv. Nonlinear Stud., 17, (3) (2017), 611-640.

[32] J. XIE, X. HUANG AND Y. CHEN, Existence of multiple solutions for a fractional p-Laplacian system with concave-convex term, Acta Math. Sci., 38B, (6) (2018), 1821-1832.

[33] S. YAN, J. YANG AND X. YU, Equations involving fractional Laplacian operator: compactness and application, J. Funct. Anal., 269, (1) (2015), 47-79.

[34] Y. YANG, The Brezis-Nirenberg problem for the fractional p-Laplacian involving critical HardySobolev exponents, preprint, https://arxiv.org/abs/1710.04654v1.

[35] J. Zhang AND T.S. HSU, Nonlocal elliptic systems involving critical Sobolev-Hardy exponents and concave-convex nonlinearities, Taiwan J. Math, (2019), (Preperint), doi:10.11650/tjm/190109. 